\title{
Analysis of End-to-End Packet Delay for Internet of Things in Wireless Communications
}

\author{
Imane Maslouhi ${ }^{1}$ \\ Dept: Electronics, Informatics and \\ Telecommunications \\ ENSAO, Mohammed I University, \\ Oujda, Morocco
}

\author{
El Miloud Ar-reyouchi ${ }^{2}$ \\ Dept. Telecommunications and \\ Computer science \\ Abdelmalek Essaadi University and \\ SNRT, Tetouan, Morocco
}

\author{
Kamal Ghoumid ${ }^{3}$, Kaoutar \\ Baibai $^{4}$ \\ Dept. Electronics, Informatics and \\ Telecommunications, ENSAO, \\ Mohammed I University \\ Oujda, Morocco
}

\begin{abstract}
Accurate and efficient estimators for End to End delay (E2EPD) plays a significant and critical role in Quality of Service (QoS) provisioning in Internet of Things (IoT) wireless communications. The purpose of this paper, on one hand, is to propose a novel real-time evaluation metrics, on the other hand, addresses the effects of varying packet payload (PP) size. These two objectives rely on the analysis of E2EPD for QoS provisioning in multi-hop wireless IoT networks through multiple hops count from source to destination. The results of this study show the critical effect of PP size, hops count and interface speed on the improving E2EPD use of applications requiring real-time IoT communications.
\end{abstract}

Keywords-End to end delay; internet of things; multi hop; wireless communication

\section{INTRODUCTION}

End-to-End delay is the time taken by a packet to travel from source to destination [1], [2] ,[3]. It is an important design and performance characteristic of IoT wireless communications networks. It is especially important for delaysensitive applications and for which need transmitting packet data with average delay constraints [4]. E2ED is a common term in IP network monitoring and differs from Round-Trip Time (RTT) [5].

The large turnout of real-time communication to the IoT gives much importance to improve E2ED. Reducing delay metric is exposed in different contexts such as access delay in [6].Delay improvement for the remote management of renewable energy using a random $\mathrm{NC}$ is also evaluated according to [7]. The evaluations of average E2ED and jitter in wireless tele-ultrasonography medical systems has been carried in [8].

E2E delay depends on number of hops in the path, congestion on the network and it is affected by various parameters as interface speed in intermediate nodes [9].

In IoT, an excessive E2E delay can significantly affect throughput. Higher delays could result in rejecting the packets by routers due to breaching the limit of Time to Live (TTL), then Internet Control Message Protocol (ICMP) packets are sent to the source and hence results in re-transmissions. E2E delay is also infected directly by the retransmission timeout
(RTO) as [10]. IoT can also strongly secure the intelligent networks platforms which is studied in [11].

The E2EPD is especially important for delay-sensitive applications. Packets are delivered to destination nodes with delays, which may vary from packet to packet (one measure is jitter). E2EPD distribution calculation method it is an analytical model to calculate E2E delays in packet networks according to [12]. Recently, simulation results demonstrate the accuracy and effectiveness of analytical E2EPD modeling for achieving delay aware as in [13].

TCP (Transmission Control Protocol) is more widely used protocol on the Internet because of their errors correction. UDP (User Datagram Protocol) is another more frequently used protocol on the Internet. However, UDP is never used to transmit valuable data such as database information, webpages, etc. UDP is commonly used for streaming audio and video. Therefore, UDP is characterized by high-speed data communication. In IoT, protocol specification allows interoperability among things with different communication standards as CTP (Communication Things Protocol) according to [14]. Other study gives an overview of some technical details that pertain to the IoT enabling technologies applications as [15].

In the Remote Management field popularity of IoT is increasing day by day in the area of remote monitoring system as in [16]. The remote monitoring systems include, remote satellite monitoring, DVB stream management, data acquisition in remote areas, energy grid monitoring etc., In IoT network management, the real-world objects communicate with each other using source-destination which source and destination can be Supervisory Control and Data Acquisition (SCADA) and remote terminal unit (RTU) respectively.

The efficiency of applying the delay distribution from a single node and using convolution to find the E2E delay is given in [17]. The E2ED distribution in a linear network is derived for homogeneous networks as in [18].

This paper gives a simplistic overview of the role that can play the payload length (based on some basic parameters) for improving the E2E delay in IoT network performance.

The rest of this paper is structured as follows. Section II discusses the general conception of payload transmission. 
Section III gives a general description of end-to-end packet delay in wireless multi-hop network, and its mathematical model is described. The experimental and simulation setup are given in Section IV. Section V lists results and discussion .In Section $\mathrm{V}$, the authors provide the conclusions.

\section{GENERAL CONCEPT}

\section{A. Transmission Average Message Size}

The basic structure of a packet varies between protocols, a typical packet includes two sections a header and payload. In this paper, the authors focus on transmitting the average message which vary in length from o to 1500 bytes, and their headers vary in complexity from five to 50 bytes. The E2ED in IoT network can be strongly dependent of the message size.

Data transmission over an E2E (source to destination) communication channel is being carried within a packet does not integrate the overhead data. These real data are referred as the payload.

For a communications layer that requires some of the overhead data to do its operation, the payload is sometimes designed to include the part of the overhead data. However, in this operational network, the payload is the bits that get delivered to the source (SCADA) at the destination( RTUn).

The most important factors that directly influence $\mathrm{E} 2 \mathrm{E}$ delay are the arrival rate, the service rate, the number (count) of hops in a path, Ethernet interface speed and the baud rate [kbps] for the serial interface (COM).

When data is sent over network and Internet connections, each IoT nodes sent incorporate both header information and the real information being sent. The header contains various things depends on the used protocol, it can detects the source and destination of the packet, while the real information is assigned to the payload. Header information is applicated singly in the transmission process, it stripped off from the packet when it has just arrived at its destination. Therefore, the payload is the only data collected by the destination IoT node. In this paper the transmission message size is compared and analyzed.

\section{B. Data Transmission Speed}

The results considered different value of the payload which is the data itself it needs to transfer (usually the user message size without any headers (IP, TCP, UDP,).

When crossing more than one device in IoT network, interface speed has always played a primordial role for improving E2E delay. It is necessary to configure the interface speed of IoT devices. In this paper it is referred to as the baud rate $[\mathrm{bps}]$ for the serial (COM) interface or an Ethernet interface speed.

In typical serial interface communication systems, the available bit rate values are: $2400 \mathrm{kbps}, 4800 \mathrm{kbps}, 9600 \mathrm{kbps}$, $19200 \mathrm{kbps}, 38400 \mathrm{kbps}$ and 115200kbps.

When using TCP instead of UDP lowers the total network capacity due to the higher TCP overhead (ETH - UDP/IP and serial options are equal. The performance evaluation of E2E delay of Randomized TCP is presented in [19].
UDP sends the packets which contains just simple things in the header as source IP/PORT and destination . TCP, on the other end contains some interesting information, namely the sequence number of the packet( to guarantee ordered delivery), a lot of flags (to guarantee the packet actually received in it's destination) and checksum of the data (to ensure it didn't get corrupted) and received correctly in its destination.

The protocols TCP and UDP are used for transmitting bits of data over the Internet. They can build on top of the IP protocol.

The packet sent directly via TCP or UDP are processed similarly, as they're forwarded from a source intermediary IoT nodes and to the destination

\section{A THEORETICAL STUDY OF END-TO-END PACKET DELAY}

The E2E delay is typically measured in multiples or fractions of seconds, in that only path in the one direction from source to destination is measured. It is very interesting to specify how long it takes for a user data size without any headers to travel across the network from source to destination.

\section{A. End to End Delay}

In telecommunication and data networks, the end-to-end packet delay $D$ usually consists of following four elements [20]:

$$
\begin{gathered}
\text { Transmission delay } D^{(t)} \text {, Radio propagation delay } D^{(r)}, \\
\text { Signal processing delay } D^{(s)} \text { and Queueing delay } D^{(q)} .
\end{gathered}
$$

Their mathematical relation can be simply expressed as

$D=D^{(t)}+D^{(r)}+D^{(s)}+D^{(q)}$

The E2E delay equation above describes the packet delay at a single IoT node along its path from source to destination.

Assuming that the radio transmission delay $D^{(t)}$ and signal processing $D^{(s)}$ delay are small enough to neglect, (1)

becomes

$D=D^{(t)}+D^{(q)}$

In the IoT connections, if $n$ is the number of IoT nodes in the connected path from source to destination, the number of links is $n+1$, the end to end delay is

$$
D_{n}=(n+1)\left(D_{n}^{(t)}+D_{n}^{(q)}\right)
$$

Where $n$ are the nodes can be considered for measuring the end-to-end delay.

\section{B. Network Model and Performance}

Consider the problem of analyzing the E2E delay over the paths from a node source $S$ to a node destination $T$ in a complete IoT network. The following Fig.1 shows an Internet of Things Network model. 


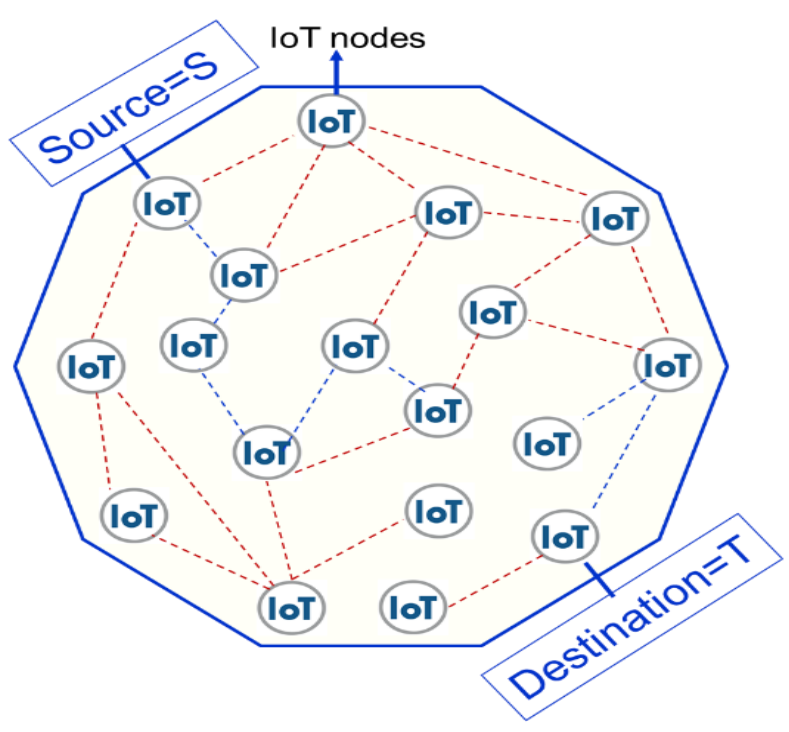

Fig. 1. Internet of Things Network model.

Assume that the signal processing delay $D_{n}^{(s)}$, in the IoT node, and Radio propagation delay $D_{n}^{(r)}$ are equal to 0 .

Suppose Source $S$ begins to transmit the first packet at $D_{(t=0)}=D_{0}$ delay over a path with $n$ IoT nodes. This packet is received by node $T$ at $D_{0}+D_{n}^{(t)}$, where $D_{n}^{(t)} \quad$ is the transmission delay of a packet.

The packet is re-transmitted to the destination $\mathrm{S}$ across $n$ IoT nodes, while he next packet is transmitted on a same path.

With $n$ IoT nodes, the first packet $P_{1}$ reaches destination node $T$ at:

$(n+1) D_{n}(t)$

If $p$ is the number of packets, the last packet sent to:

$(p-1) D_{n}(t)$

The last packet arrives at (which corresponds to the end of the transfer):

$(p-1) D_{n}+(n+1) D_{n}$

Either again,

$D_{n}(t)(p+N)$

By posing $D_{n}(t)=L / p R$, where $p$ and $R$ are the number of packets and data rate respectively, the crossing delay of the network $D_{n}$ is obtained as :

$D_{n}=(L / p R)(p+N)$

Or

$D_{n}=(L / R)(1+N / p)$

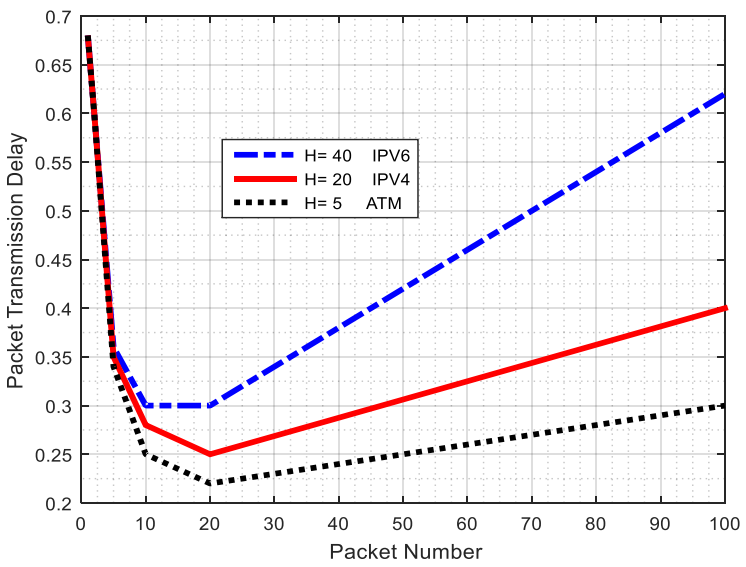

Fig. 2. Evolution of Packet Transmission Delay vs Packet Number.

There however, this formula does not consider the protocol data $(\mathrm{H})$, Which should be added to each packet, hence:

$T_{p}=\left(\frac{L+p H}{R}\right)\left(1+\frac{N}{p}\right)$

The curves given in Fig.1graphically illustrates the theoretical result with the following hypothesis: $\mathrm{L}=1500$ bytes, $\mathrm{N}=5$

The packet transmission delay increases significantly versus of packet number. The values are expressed depending on the message switching ( $p=1$ and $n \geq 0$ ) that is a network switching technique in which data is routed in its entirety from the source $S$ to the destination $T$.

\section{If $p=1, n \succ 0$ Message Switching \\ if $p=1, n=0$ Circuit Switching}

Fig.2 compares the performance according to a header: ATM ( 5 bytes), IPV4 (20 bytes), and IPV6 (40 bytes).

The transmission delay, in the network, is even lower than is small. This leads to search for routes that minimize the number of nodes crossed (routing algorithms) and to increase the network (increase the probability of finding a more direct route).

It should be noted that the influence of the service header size is not negligible.

\section{EXPERIMENTAL AND SIMULATION SETUP}

In this practical application, the topology showed in Fig. 1 is well-respected. This paper studies the E2ED of an IoT wireless network, the system is configured as a single source $S$ node sends packets to single destination $T$ node across several IoT nodes.

The IoT Network system consists of two parts, related hardware and management software. The system hardware is divided into on source node, wireless transmission IoT nodes and destination node. The software adopts a centralized control management model, providing users basic information management for real-time monitoring, 
TABLE I. SIMULATION PARAMETERS

\begin{tabular}{|l|l|}
\hline Critical parameters & Value \\
\hline Packet Payload [bytes] & $0-1500$ \\
\hline Processing time[ms] & 20 \\
\hline Interface speed [kbps] & $2400,38400,115200$ \\
\hline Hops & $1-9$ \\
\hline ACK & off \\
\hline
\end{tabular}

The SunSet E20c is a device used to measure E2E delay which provides a full transmission testing according to [21]. It can also verify Datacom circuits by monitoring the received information, control leads, and physical layer results.

The simulation results were developed using Matlab software.

Data transfer rate and interface speed of each IoT nodes are same as IoT nodes wireless communication module. If more IoT nodes are used in E2E delay path it will increase and it performance can improved by reducing the packets size as in [22].

Table I gives some simulation parameters used for the analysis of E2E listing conditions selected.

\section{RESULTS AND DISCUSSION}

This section describes the experiments and simulations results. The presented results illustrate how E2E delay (between $S$ and $T$ ) varies depending on the PP for several hops and UMS (100 Bytes and 1500 Bytes) of wireless edges in wireless communication for IoT, then compare the simulation result between Ethernet TCP/IP and UDP/IP as interface speed using fixed values of PP of multi-hop wireless IoT networks.

Fig.3 reveals the E2E delay measurements as a function of hops count per static path for different sizes of data without any headers. It also shows the multi hop transmission from a source to destination through IoT nodes.

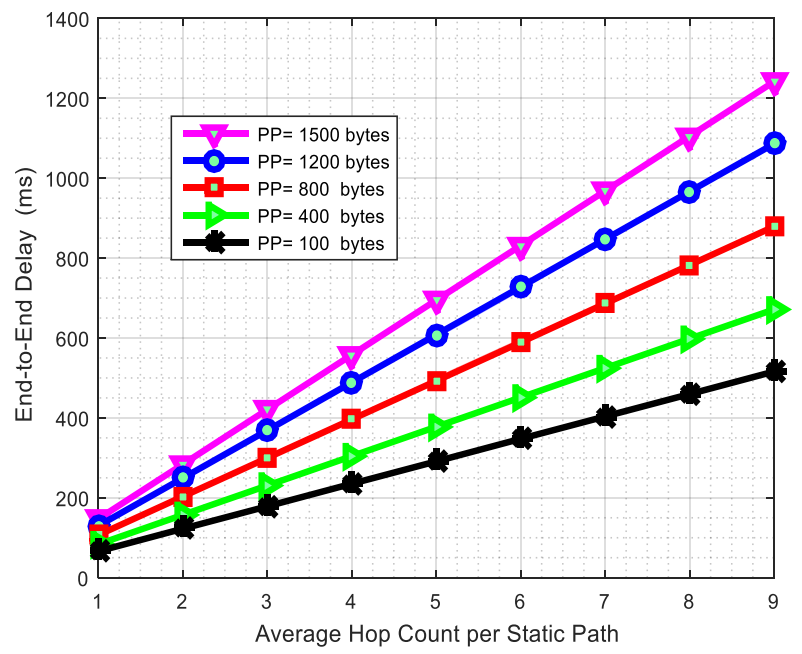

Fig. 3. End to End Delay vs Hop Count Per Static Path.
TABLE II. END TO END DELAY RESUlt OF MULTI-HOP PATHS

\begin{tabular}{|c|l|l|l|l|}
\hline \multirow{2}{*}{$\begin{array}{c}\text { PP } \\
\text { bytes }\end{array}$} & \multicolumn{4}{|c|}{ Hops } \\
\cline { 2 - 5 } & 1 & 3 & 6 & 9 \\
\hline 100 & 66 & 178.5 & 347.5 & 516 \\
\hline 800 & 106.5 & 299.5 & 589 & 879 \\
\hline 1500 & 147 & 420 & 831 & 1241 \\
\hline
\end{tabular}

For tree, six and nine hops transmission, the average E2ED is around $420 \mathrm{~ms}, 831 \mathrm{~ms}$, and $1241 \mathrm{~ms}$ for the maximum payload offered ( $\mathrm{PP}=1500$ bytes) respectively, while $178 \mathrm{~ms}$ ,347 ms, and $516 \mathrm{~ms}$ respectively for $\mathrm{PP}=100$ bytes.

It is observed that the respective E2ED were linearly increased with increasing hops count or packet payload.

Table II below summarizes some of the simulation results.

Adding one more hop in transmission path increases the E2E delay by $53.878 \%, 52.463 \%$ and $51.852 \%$ for 100 bytes, 800 bytes and 1500 bytes respectively. Consequently, each of those hops introduces some delay according to a payload size.

Fig.4 illustrates the results of indirect transmissions through IoT nodes over 9 hops for different packet payload size and give details of how E2E delay varies in terms of different interface speed (2400kbps up to $115200 \mathrm{kbps}$ ) of nine hops.

Results indicated in Fig.4 interface speed has a direct and significant effects on E2ED.

The $\mathrm{PP}=0$ bytes in Fig .4 means that data size is zero because the packet is only acknowledging data; it is not transmitting any data. Packets with an ACK flag and 0 size can be TCP keep alive packets. There are other circumstances in which a system will send TCP packets with zero length.

The Table 3 below represents some simulations results of E2E delay using the baud rate [kbps] for the serial (COM) interface (three different payload sizes for a path with 9 hops).

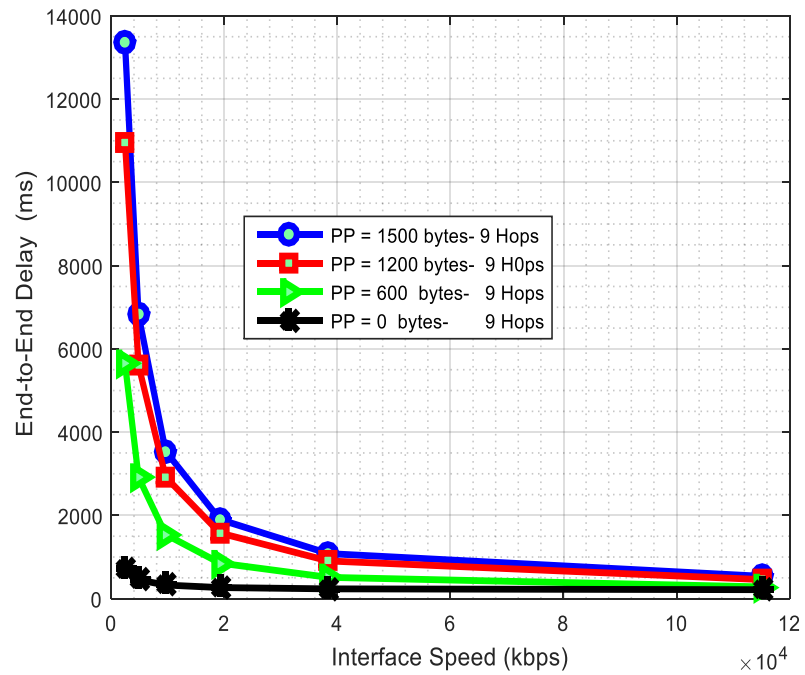

Fig. 4. End to End Delay vs Interface Speed. 
TABLE III. E2E DELAy RESUlts OF CRITICAL INTERFACE SPEED

\begin{tabular}{|l|l|l|l|}
\hline \multirow{2}{*}{ PP bytes } & \multicolumn{3}{|c|}{ Interface speed } \\
\cline { 2 - 4 } & 2400 & 38400 & 115200 \\
\hline 0 & 347 & 117 & 107 \\
\hline 600 & 5658 & 511 & 282 \\
\hline 1500 & 13370 & 1082 & 535 \\
\hline
\end{tabular}

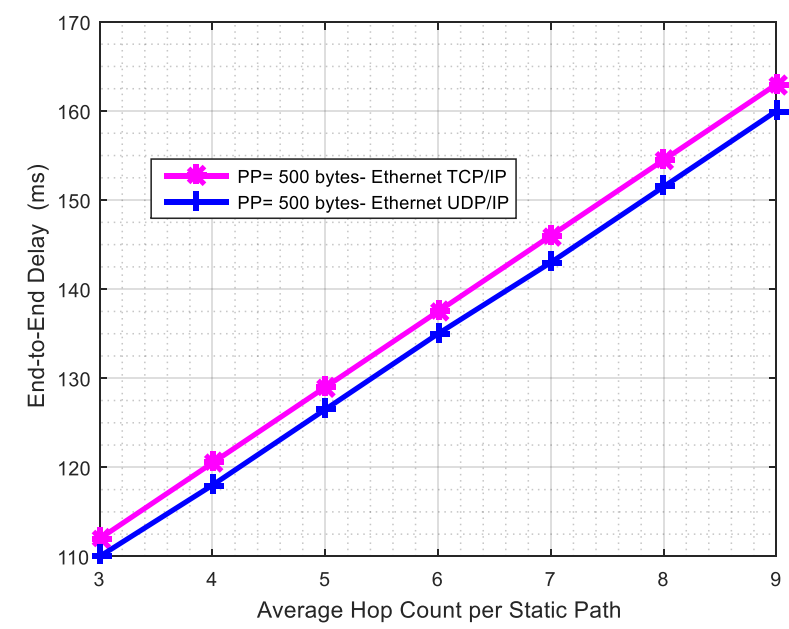

Fig. 5. End to End Delay vs Hop Count Per Static Path using ETH TCP/IP and ETH UDP/IP.

From this table it can be seen that the more the interface speed increase, the more there is improvement of E2E delay.

Fig. 5 shows experimental results of the comparison between Ethernet TCP/IP and UDP/IP as interface speed results for $\mathrm{PP}=500$ bytes.

Fig.5 illustrates that the UDP is speedily than TCP. The reason is because there is no form of flow control or error correction or its absent acknowledge packet (ACK) that allows a continuous packet flow, instead of TCP that acknowledges a determined packets.

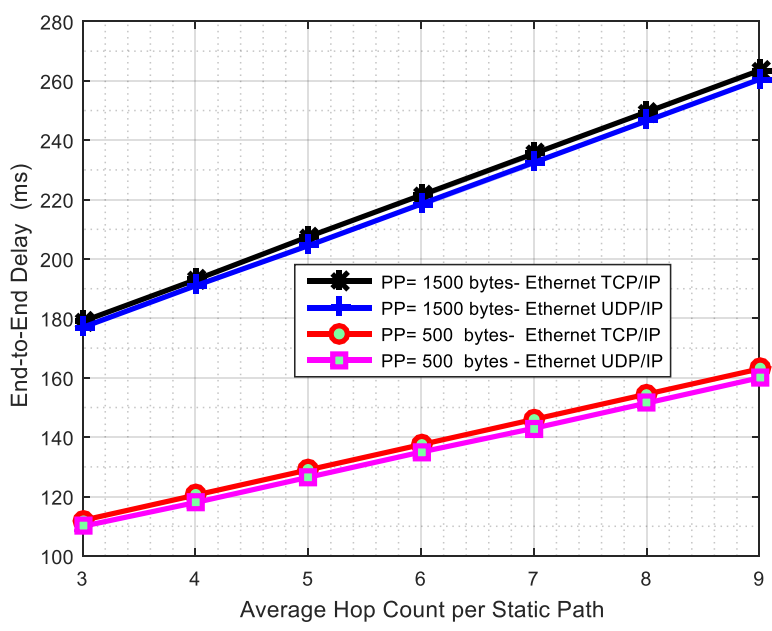

Fig. 6. Comparison End to End Delay vs Hop Count Per Static Path ( $\mathrm{PP}=500$ Bytes and 1500bytes).
When using UDP, packets are just sent to the recipient. The sender continues transmitting the next packets (without waiting that the recipient received of the previous packet) If the IoT destination misses a few UDP packets, they are lost. The sender will not resend them. Losing all this overhead means the IoT nodes can communicate more speedily.

Fig. 6 shows simulation result of the comparison between Ethernet TCP/IP and UDP/IP as interface speed using two values of PP (500 bytes and 1500 bytes).

Table 4 lists the comparison of the average delay from simulation and estimation results using Ethernet interface speed.

It can also be further concluded that the values developed performs well under different conditions.

TCP/IP is a suite of protocols used by IoT nodes to communicate over the Internet. UDP/IP is used by applications to deliver a speedily stream of information by doing away with error-checking.

In this paper, the analysis of E2E packet delay for internet of things in wireless communications was developed and illustrated by both experimental and simulations results. This analysis, compared to the analysis results, gives more a simplistic and quickly overview for improving the E2E delay in IoT network performance as in [17].

Consider the links from node 1 to node 10, keeping the same assumptions of [12]. For $2 \mathrm{Mbit} / \mathrm{s}$ links and constant packet lengths of 400 bytes, the comparison between the E2EPD distribution calculation method given by [12] and the proposed method clearly shows the efficiency and accuracy of the results of E2EPD obtained by the proposed method as in Fig.7.

TABLE IV. COMPARISON OF E2E DELAY USING TCP/IP AND UDP/IP

\begin{tabular}{|c|c|c|c|c|}
\hline \multirow{2}{*}{$\begin{array}{c}\text { PP } \\
\text { (bytes) }\end{array}$} & \multicolumn{2}{|c|}{ 4 Hops } & \multicolumn{2}{c|}{ 8 Hops } \\
\cline { 2 - 5 } & $\mathrm{TCP} / \mathrm{IP}$ & $\mathrm{UDP} / \mathrm{IP}$ & $\mathrm{TCP} / \mathrm{IP}$ & $\mathrm{UDP} / \mathrm{IP}$ \\
\hline 500 & 120 & 118 & 154 & 151 \\
\hline 1500 & 193 & 191 & 249 & 246 \\
\hline
\end{tabular}

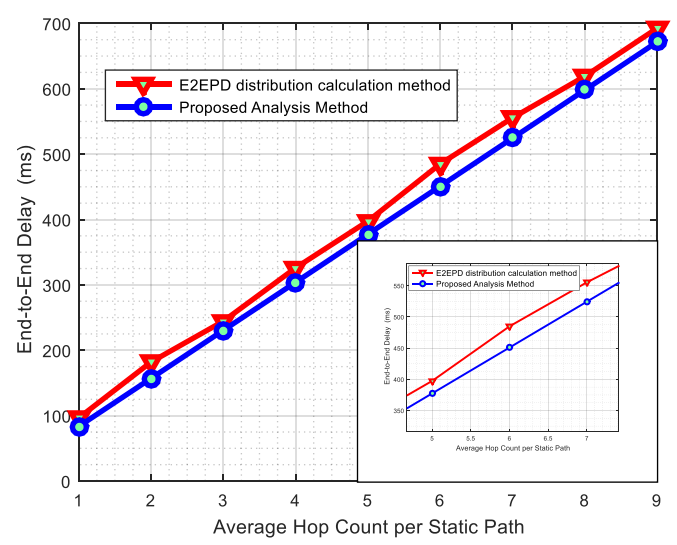

Fig. 7. Comparison between the E2EPD Distribution Calculation Method and the Proposed Method.( $\mathrm{PP}=400$ bytes ). 
It was also observed (in Fig.7 with zoomed curves) that the respective E2EPD distribution calculation method does not increase linearly with increasing hops count or packet payload while the E2EPD of the proposed method increase in straight line. The simple and the efficient proposed method for analyzing E2ED gives a very accurate and robust results.

\section{CONCLUSIONS}

The authors have performed both an experimental and theoretical analysis of the End to End delay which is influenced by critical parameter. The simulation, measurement and estimation results were shown the impacts of payload size, hops count and interface speed on E2E delay performance. The results showed that this estimator provides good estimates of payload packets, End to end delay, and jitter gave a key insight into the QoS provisioning for multi-hop wireless networks. Ethernet UDP/IP is exploited when a speed is requested and error correction is not needful. The setting parameters discussed in this paper gives a rapid and easy idea of the E2E delay improvement in the IoT network. The E2EPD for narrowband Internet of Things in Wireless Communications nodes should be analyzed and minimized finding new techniques and methods which will be the future works.

\section{ACKNOWLEDGMENT}

This work was partially supported by the SNRT (laboratory of regional service) Morocco.

\section{REFERENCES}

[1] S.-N. Yeung and J. Lehoczky, "End-to-end delay analysis for real-time networks," in Proc. IEEE RTSS, London, U.K., Dec. 2001, pp.299-309

[2] El.M..AR-REYOUCHII, K. Ghoumid, K. Ameziane, and O. El Mrabet, "The Improvement of End to End Delays in Network Management System Using Network Coding," International Journal of Computer Networks \& Communications (IJCNC), vol.5,n 6 (Nov. 2013), :65-84. DOI: $10.5121 / \mathrm{ijcnc} .2013 .5604$.

[3] Nasim Abbas; Fengqi Yu ,'Performance analysis of end-to-end delay and reliability for linear wireless multimedia sensor networks ," IEEE 3rd Information Technology and Mechatronics Engineering Conference (ITOEC), 3-5 Oct. 20172017

[4] R. A. Berry and R. Gallager, "Communication over fading channels with delay constraints," IEEE Trans. Inform. Theory, vol. 48, May 2002, pp. $1135-1148$.

[5] El. .Ar-Reyouchi, K. Ghoumid, K.Ameziane, O. El Mrabet,"Performance Analysis of Round Trip Time in Narrowband RF Networks For Remote Wireless Communications," International Journal of Computer Science \& Information Technology (IJCSIT), vol.5,n 5, October 2013,pp.1-20. 10.5121/ijcsit.2013.5501

[6] L.Zhao ; W. Sun ; Y. Shi ; J. Liu," Optimal Placement of Cloudlets for Access Delay Minimization in SDN-Based Internet of Things Networks", IEEE Internet of Things Journal,vol.5,n..2(2018), 1334 1344 DOI: $10.1109 /$ JIOT.2018.2811808
[7] El .Ar-reyouchi,M.Hammouti,I. Maslouhi, K.Ghoumid "The internet of things: Network delay improvement using network coding," ACM ,ICC ,Proceedings of the Second International Conference on Internet of things, Data and Cloud Computing, Cambridge, United Kingdom, ,March 22 - 23, 2017 . DOI: 10.1145/3018896.3018902

[8] Y. Chen, N. Philip, R. Istepanian, and I. Darwazeh, "End-to-end delay distributions in wireless tele-ultrasonography medical systems," in Global Telecommunications Conference (GLOBECOM 2013), 2013 IEEE, 2013.

[9] El. Miloud Ar Reyouchi, K.Ghoumid, K. Ameziane, O. El Mrabet, S.Mekaoui," Performance Analysis of Round Trip Delay Time in Practical Wireless Network for Telemanagement," World Academy of Science, Engineering and Technology International Journal of Electronics and Communication Engineering,vol7, n.11( 2013). 14131419. scholar.waset.org/1999.5/17295.

[10] E.G. Davis, A. Calveras and I. Demirkol, "Improving packet delivery performance of publish/subscribe protocols in wireless sensor networks," Sensors, 2013,vol. 13, no. 1, pp. 648-680.

[11] Maslouhi I., Ghoumid K., Zaidouni J., Benzekri W., Ar-reyouchi E.M., Lichioui A. (2016) ,"Network Higher Security of Intelligent Networks Platforms in Telecommunications Areas,". In: Proceedings of the Mediterranean Conference on Information \& Communication Technologies 2015. Lecture Notes in Electrical Engineering, vol 380. Springer.

[12] O. Osterbo, "Models for calculating end-to-end delay in packet networks," ITC-18, Berlin, Germany, August 31-September 5, 2003, pp. 1231-1240.

[13] Qiang Ye,; Weihua Zhuang, Xu Li , Jaya Rao, "End-to-End Delay Modeling for Embedded VNF Chains in 5G Core Networks," IEEE Internet of Things Journal,2018 ,DOI: 10.1109/JIOT.2018.2853708.

[14] A.Asensio,A. Marco, R. Blasco and R. Casas, "Protocol and Architecture to Bring Things into Internet of Things," International Journal of Distributed Sensor Networks, 2014.

[15] A. Al-Fuqaha, M. Guizani, M. Mohammadi, M. Aledhari, and M. Ayyash, "Internet of things: A survey on enabling technologies, protocols, and applications," IEEE Communications Surveys Tutorials, vol. 17, no. 4, pp. 2347-2376, 2015.

[16] El Miloud Ar Reyouchi, "Optimisation des performances des réseaux de communications sans fil : Performances des réseaux de communications sans fil pour la télégestion des stations de la Télédiffusion TV/FM ," Paf, ,2017, ISBN 978-3-8416-4286-8.

[17] R. Stewart,"End-to-end Delay analysis for Small/Medium Scale IP networks," PhD Thesis, University of London 2003.

[18] M. Xi and M. Haenggi, "Towards an end-to-end delay analysis of wireless multihop networks," Ad Hoc Netw., vol. 7, no. 5, pp. 849-861, Jul. 2009

[19] M.Shorfuzzaman, M.Masud and Md. M. Rahman,"Characterizing Endto-End Delay Performance of Randomized TCP Using an Analytical Model," International Journal of Advanced Computer Science and Applications(IJACSA), 2016. Vol.7,n.3, 2016.

[20] D. Bertsekas and R. Gallager, Data Networks. Prentice-Hall, 1987.

[21] Y.Chatei,M.Hammouti, .E.M. Ar Reyouchi, and K. Ghoumid,"Downlink and Uplink Message Size Impact on Round Trip Time Metric in Multi-Hop Wireless Mesh Networks,"vol.8, 2017/03/01DOi - 10.14569/IJACSA.2017.080332.

[22] G. Hasegawa, T. Tomioka, K. Tada, and M. Murata, "Simulation studies on router buffer sizing for short-lived and pacing TCP flows,,"Computer Communications, vol. 31, no. 16, pp. 3789 - 3798, 2008. 